\title{
Apoptosis induction in BEFV-infected Vero and MDBK cells through Src-dependent JNK activation regulates caspase- 3 and mitochondria pathways
}

\author{
Chun-Yen $\mathrm{CHEN}^{1 \dagger}$, Chin-Yang $\mathrm{CHANG}^{1 \dagger}$, Hung-Jen Liu ${ }^{2,3}$, Ming-Huei LiaO ${ }^{2,3}$, \\ Chi-I $\mathrm{CHANG}^{2}$, Jue-Liang $\mathrm{Hsu}^{2}$, Wen-Ling $\mathrm{SHIH}^{2 *}$
}

\footnotetext{
${ }^{1}$ Graduate Institute and Department of Life Science, Tzu-Chi University, Hualien, Taiwan, Republic of China

${ }^{2}$ Graduate Institute of Biotechnology, National Pingtung University of Science and Technology, 1, Shuefu Rd., Neipu, Pingtung, 91201, Taiwan, Republic of China

${ }^{3}$ Department of Veterinary Medicine, National Pingtung University of Science and Technology, Pingtung, Taiwan, Republic of China
}

\begin{abstract}
Our previous report demonstrated that bovine ephemeral fever virus (BEFV)-infected cultured cells could induce caspase-dependent apoptosis. This study aims to further elucidate how BEFV activates the caspase cascade in bovine cells. BEFV replicated and induced apoptosis in Vero and Madin-Darby bovine kidney (MDBK) cells, and a kinetic study showed a higher efficiency of replication and a greater apoptosis induction ability of BEFV in Vero cells. Src and c-Jun N-terminal kinase (JNK) inhibitor, but not extracellular signal-regulated kinase (ERK) or p38 inhibitor, alleviated BEFV-mediated cytopathic effect and apoptosis. In BEFV-infected Vero and MDBK cells, BEFV directly induced Src tyrosine-418 phosphorylation and JNK phosphorylation and kinase activity, which was inhibited specifically by SU6656 and SP600125, respectively. The caspase cascade and its downstream effectors, Poly (ADP-ribose) polymerase (PARP) and DFF45, were also activated simultaneously upon BEFV infection. In addition, cytochrome c, but not Smac/DIABLO, was released gradually from mitochondria after BEFV infection. SU6656 suppressed Src, JNK, and caspase-3 and -9 activation, as well as PARP and DFF45 cleavage; SP600125 reduced JNK and caspase-3 and -9 activation, as well as PARP and DFF45 cleavage. Taken together, these results strongly support the hypothesis that a Src-dependent JNK signaling pathway plays a key role in BEFV-induced apoptosis. The molecular mechanism identified in our study may provide useful information for the treatment of BEFV.
\end{abstract}

BEFV / apoptosis / caspase / Src / JNK

\section{INTRODUCTION}

Bovine ephemeral fever (BEF), also known as three-day sickness, is an arthropod vectorborne disease. Although mortality is usually low, cattle in good condition are affected more severely: mortality rates as high as $30 \%$ have been reported in very fat cattle. BEF is an eco-

\footnotetext{
$\dagger$ The first two authors contributed equally to this work.

*Corresponding author: wlshih@mail.npust.edu.tw
}

nomically-important viral disease of cattle and buffalo that is caused by the bovine ephemeral fever virus (BEFV), a member of the genus Ephemerovirus of the Rhabdoviridae family. $\mathrm{BEF}$ is endemic in most tropical and subtropical areas of Africa, Australia, the Middle East, and Asia $[28,34]$. BEFV-infected cattle with excessive nasal discharge and a protruding tongue as a result of dyspnea were observed in Taiwan in August 1996, and most of the affected cattle were seen to be difficult to treat and had a poor prognosis. A vaccination regimen was initiated

This is an Open Access article distributed under the terms of the Creative Commons Attribution-Noncommercial License (http://creativecommons.org/licenses/by-nc/3.0/), which permits unrestricted use, distribution, and reproduction in any noncommercial medium, provided the original work is properly cited. 
in 1984. Previous reports have indicated that, before vaccination, $94 \%$ of the animals studied were already seropositive, which is suggestive of an endemic or persistent infection from the previous year, and that vaccine-induced immunity was partially protective against BEF $[15$, $20,39]$. In terms of pathology, the inflammatory response of infected animals can be blocked by the administration of anti-inflammatory drugs, which can prevent clinical signs such as fever and viremia, and further reduce the frequency of viral isolation in the animals [37]. In numerous in vitro studies, viral infection can directly induce apoptosis without the involvement of immune cells [7]; however, the pathogenic mechanism in vivo is more complicated for virus-induced diseases. Studies of animal models have shown that the apoptosis process can facilitate viral clearance, but may also cause virus-induced tissue injury and resulting disease [7]. Since viral infection may produce a different tissue- or host-specific response, the detailed mechanism in several virus-host systems remains unclear and needs to be investigated.

Evidence indicates that infection by rhabdoviruses such as the vesicular stomatitis virus (VSV), spring viremia of carp virus (SVCV), and the rabies virus, as well as BEFV, results in apoptotic cell death $[5,21]$. The mechanism of VSV-induced apoptosis requires caspase cascade activation and suppression of host gene expression, and its Matrix protein plays a major role in mediating the apoptotic process $[8,11$, 14]. Interestingly, VSV can replicate in tumor cells selectively to induce apoptosis of tumor cells [12, 27]. Study of VSV-encoded matrix protein revealed anti-tumor and anti-metastatic activities, as well as improvement following chemotherapy and radiation therapy in several tumor cell lines and animal models [9, 31, 32, 42]. The cytopathic effect (CPE) is caused by apoptosis, which is damage to infected host cells caused by virus infection, and leads to visible morphologic changes. Different types of cells in a host individual may respond differently to a viral infection. Study of BEFV has shown that viral protein synthesis can be detected $12 \mathrm{~h}$ after BEFV infection at an early stage of CPE, and the process can last for more than $12 \mathrm{~h}$ post-infection (hpi) [38]. Therefore, the study of the underlying mechanism of virus-induced apoptosis may reveal a way in which to reduce the $\mathrm{CPE}$ and thereby reduce the mortality caused by BEFV infection.

Our previous investigation demonstrated that BEFV induces apoptosis in several cell lines. It has also been shown that apoptosis can be blocked by the caspase inhibitor Z-VAD-fmk, indicating that BEFV induces caspase-dependent apoptosis in cultured cells [5]. However, the possible signaling involved in the modulation of caspase activation in BEFV-infected bovine cells has not been studied in detail. Current data indicate that BEFV activates Src and c-Jun N-terminal kinase (JNK) signaling and then subsequently regulates cytochrome c release from mitochondria and caspase activation in Vero and Madin-Darby bovine kidney (MDBK) cells. This further suggests that BEFV could influence and modulate the cellular response. The results obtained strongly support our hypothesis and are presented herein.

\section{MATERIALS AND METHODS}

\subsection{Antibodies and reagents}

Src phospho-tyrosine 418 antibody was purchased from Upstate Biotechnology (Lake Placid, NY, USA); all other antibodies and the JNK activity assay kit used in this study were purchased from Cell Signaling Technology (Danvers, MA, USA). All inhibitors were purchased from Calbiochem (EMD, Madison, WI, USA). Propidium iodide, MTT (Thiazolyl Blue Tetrazolium Bromide), protease inhibitor cocktail, and phospohatase inhibitor cocktail were obtained from Sigma (St. Louis, MO, USA). A cytosol and mitochondria fractionation assay kit was obtained from Pierce (Rockford, IL, USA).

\subsection{Virus, cell culture, and MTT assay}

The PT-1 strain BEFV isolate was kindly provided by Dr Ming-Huei Liao (National Pingtung University of Science and Technology). Virus titers were determined by plaque assay [36]. Confluent cells on a $3.5-\mathrm{cm}$ plate were infected with 10 -fold dilutions of virus in $0.2 \mathrm{~mL}$ of media and were incubated for $1 \mathrm{~h}$ at $37{ }^{\circ} \mathrm{C}$ to allow virus adsorption. The cells were then washed to eliminate any unbounded virus; $2 \mathrm{~mL}$ of medium were subsequently added and the cultures 
incubated at $37{ }^{\circ} \mathrm{C}$ for 3 days. The monolayers were then fixed with a $10 \%$ formaldehyde solution in phosphate-buffered saline and stained with $0.25 \%$ crystal violet in order to visualize the virus plaques. Individual foci were obvious and plaques were enumerated on plates, all dilutions being plated in duplicate. Titers were expressed as mean plaque-forming units per $1 \mathrm{~mL}$ (pfu/mL). Vero (African green monkey kidney cells) and MDBK cells were cultured in Dulbecco's modified Eagle's medium containing 10\% fetal bovine serum, $50 \mu \mathrm{g} / \mathrm{mL}$ streptomycin, $50 \mathrm{U} / \mathrm{mL}$ penicillin, and $1.25 \mu \mathrm{g} / \mathrm{mL}$ fungizone in a humidified $37^{\circ} \mathrm{C}$, $5 \% \mathrm{CO}_{2}$ incubator. The MTT assay was performed to evaluate the proliferation of viable cells [3]. Briefly, 5000 cells were placed in each well of a 96-well plate with $200 \mu \mathrm{L}$ medium per well, and after specific treatment, $20 \mu \mathrm{L}$ MTT solution was added at a concentration of $5 \mathrm{mg} / \mathrm{mL}$, followed by incubation $\left(37^{\circ} \mathrm{C}, 5 \%\right.$ $\mathrm{CO}_{2}$ ) for $4 \mathrm{~h}$ to allow the MTT to be metabolized. The medium was then removed and cells were resuspended in formazan in $200 \mu \mathrm{L}$ DMSO. The Optical Density (OD) was read at $560 \mathrm{~nm}$, and was found to be directly correlated with cell quantity, as expected.

\subsection{Apoptosis assay}

Apoptotic ELISA and flow cytometry were utilized in this study, the experimental procedures for which have been described in our earlier reports [23, 41]. Apoptotic cell death was assessed by ELISA according to the instructions provided by Roche Applied Sciences. Briefly, $10^{3}$ cell lysates were placed into a streptavidin-coated microtiter plate and a mixture of anti-histone biotin and anti-DNA peroxidase was added. During incubation, the anti-histone antibody binds to the histone components of the nucleosomes and simultaneously the immunocomplex is captured by streptavidin via biotinylation. Additionally, the anti-DNA peroxidase reacts with the DNA components of the nucleosomes. Color was developed by addition of ABTS substrate, and the absorbance at $405 \mathrm{~nm}$ was measured. The enrichment factor was calculated by the formula: absorbance of the sample (dying/dead cells)/absorbance of the negative control. For the flow cytometery experiment, adherent and floating cells were collected by centrifugation. Pelleted cells were then washed and resuspended in PBS buffer, and subsequently permeabilized with $70 \%$ ethanol and incubated overnight at $4{ }^{\circ} \mathrm{C}$. The cells were then centrifuged, resuspended in PBS, and incubated for $1 \mathrm{~h}$ at $37^{\circ} \mathrm{C}$ with $50 \mu \mathrm{g}$ of propidium iodine $/ \mathrm{mL}$ and $100 \mu \mathrm{g}$ of $\mathrm{RNase} \mathrm{A} / \mathrm{mL}$. Aliquots of $10^{6}$ cells were analyzed using flow cytometry, and the cytosol and mitochondria fractionation assay was performed as per the manufacturer's instructions (Calbiochem, San Diego, CA, USA).

\subsection{One-step growth curve}

Cultured cells were infected with BEFV at a multiplicity of infection (moi) of 1. After a 2-h incubation period at $37{ }^{\circ} \mathrm{C}$, extracellular virions were eliminated by washing with PBS three times, and the cells were then overlaid with fresh medium. At different times post-infection, the progeny virus was plaque-titrated in MDBK cells.

\subsection{Western blotting analysis}

Vero and MDBK cells were infected with BEFV at the indicated dosage for the indicated length of time. Inhibitor treatment was performed $4 \mathrm{~h}$ before virus inoculation. Total protein was extracted by RIPA lysis buffer (150 mM sodium chloride, 1\% NP-40, $50 \mathrm{mM}$ Tris- $\mathrm{HCl} \mathrm{pH} 8.0,0.5 \%$ sodium deoxycholate, $0.1 \%$ SDS, protease or phosphatase cocktail) then quantified with the Biorad protein assay. A total of $30 \mu \mathrm{g}$ protein was subjected to SDS-PAGE, transferred to PVDF (polyvinylidene difluoride) membrane, and reacted with the indicated first antibody overnight at $4{ }^{\circ} \mathrm{C}$ with gentle shaking. The membrane was subsequently washed with TBST $(10 \mathrm{mM}$ Tris-HCl, $\mathrm{pH} 7.5$, $150 \mathrm{mM} \mathrm{NaCl}, 0.05 \%$ Tween-20), probed with the secondary antibody, washed, and then developed by addition of Western Chemiluminescent HRP Substrate (Millopore, Billerica, MA, USA).

\subsection{Statistical analysis}

All data presented were obtained from at least three independent experiments and are presented as mean \pm standard deviation (S.D.). Statistical significance was assessed by the Student's $t$-test, carried out using the software SigmaPlot.

\section{RESULTS}

\subsection{Different replication and apoptosis induction kinetics of BEFV in Vero and MDBK cells}

In order to determine the BEFV replication kinetics in Vero and MDBK cells, we initially compared the efficiency of viral replication in these two cell lines. The one-step growth curves 
shown in Figure 1A show that BEFV replicates more efficiently in Vero than in MDBK cells. The experimental conditions for the following investigation were then chosen based on this result. In order to compare the apoptosis induction kinetics by BEFV in Vero and MDBK cells, apoptotic ELISA was utilized to quantify the degree of apoptosis. Figures $1 \mathrm{~B}$ and 1C show a dose- (Fig. 1B) and time-dependent (Fig. 1C) apoptosis pattern in the two cell lines. The extent of apoptosis was correlated precisely with the BEFV-induced CPE (data not shown); however, apoptosis of infected MDBK cells was revealed to be lesser in extent than in Vero cells.

\subsection{Alleviation of BEFV-mediated apoptosis by JNK and Src inhibition}

In order to clarify whether the mitogen-activated protein kinase (MAPK) and Src pathways play significant roles in mediating this apoptotic phenomenon, we first blocked MAPK signaling by MAPK inhibitors PD98059, SB203580, and SP600125, and inhibited Src by SU6656 and C-terminal Src kinase (Csk), then we infected the cells and measured the levels of apoptosis and cell survival. The inhibitor concentrations used were not deleterious to the cells, as determined by measuring the lactate dehydrogenase activity (LDH) (data not shown). The working concentrations of the various inhibitors were as follows: $10 \mu \mathrm{M}$ PD98059, $5 \mu \mathrm{M}$ SB203580, $10 \mu \mathrm{M}$ SP600125, and $10 \mu \mathrm{M}$ SU6656 (which block extracellular signal-regulated kinase (ERK)1/2, p38, JNK, and Src, respectively). Ectopic expression of Csk was determined by Western blotting (data not shown). Figures $2 \mathrm{~A}$ (Vero cells) and 2B (MDBK cells) show that in the presence of SP600125, SU6656, and Csk, CPE induced by BEFV was dramatically suppressed, whereas inhibition of p38 and ERK1/ ERK2 had no effect. LY294002 (PI 3-kinase inhibitor) treatment was used as a control to confirm the critical roles of JNK and Src. Table I shows that the Vero and MDBK cells infected with 2 moi BEFV displayed an average of $67.5 \%$ and $23.3 \%$ apoptotic subG1 cells, respectively. Pre-treatment with SP600125 and SU6656 protected cells from BEFV-induced apoptosis. The OD value as determined by MTT assay further confirmed that inhibition of JNK and Src resulted in enhancement of cell protection and cell proliferation.

\subsection{BEFV enhanced Src tyrosine-418 phosphorylation and JNK activity}

Having demonstrated the involvement of Src and JNK, we then further evaluated their activity changes in the cells after viral infection. The results demonstrate that BEFV elicited Src tyrosine-418 phosphorylation in Vero and MDBK cells in a time-dependent (Figs. 3A, 3B, and 3C) and dose-dependent (Fig. 3D) manner, and that Src phosphorylation was suppressed in the presence of Src-specific inhibitor SU6656. Identical data were obtained by measurement of Src tyrosine-418 by ELISA (data not shown). Tyrosine- 418 of Src is located in the catalytic domain and is a site of phosphorylation. Phosphorylation of this tyrosine residue is necessary for maximum catalytic activity of Src $[2,30]$. We then further measured the activity of another critical molecule, JNK, by Western blotting, and found that JNK phosphorylation gradually increased in a time-dependent manner in Vero (Fig. 4A) and MDBK (Fig. 4B) cells upon BEFV infection. Total intracellular JNK levels were not altered. Interestingly, we routinely detected JNK1 and JNK2 expression and phosphorylation in Vero cells, whereas expression of only one JNK isoform and phosphorylation could be detected in MDBK cells. We considered that it is very likely that one dominant $\mathrm{JNK}$ isoform is present in bovine cells, and the other isoform is very likely expressed at a much lower level. Using the c-Jun fusion protein as the substrate, JNK kinase assay (Fig. 4C) demonstrated that BEFV upregulated JNK activity, and that the activation was inhibited in the presence of specific inhibitors.

\subsection{SU6656 and SP600125 block BEFV-induced caspase and DFF45 cleavage, as well as cytochrome c release from mitochondria}

To confirm the role of caspase in BEFVinduced apoptosis, a panel of antibodies was used to evaluate the status of caspase and its downstream effectors in BEFV infection. 

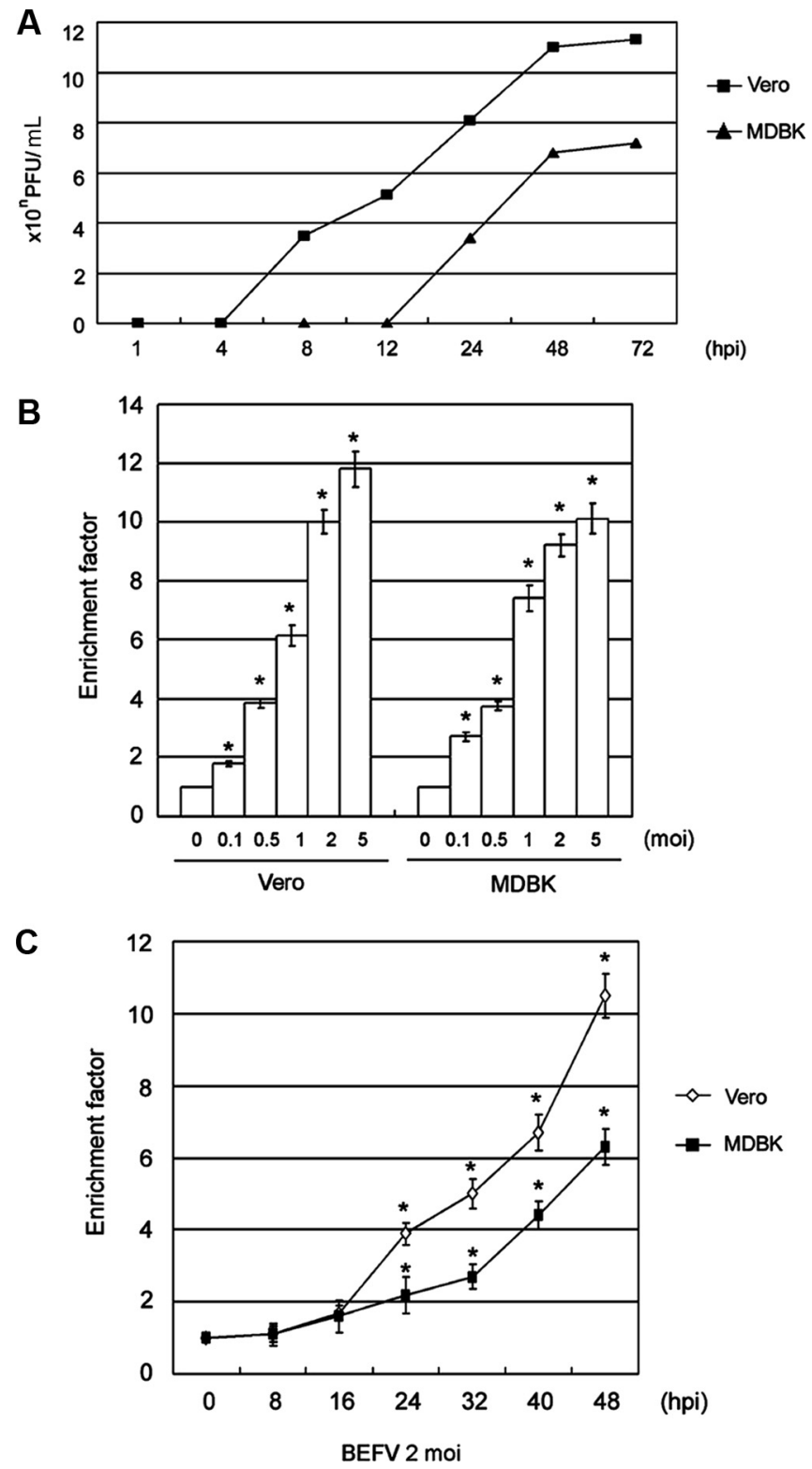

Figure 1. The different replication and apoptosis induction kinetics in BEFV-infected Vero and MDBK cells. (A) One-step growth curve analysis. Two cell lines were infected with 1 moi BEFV and then incubated for the indicated length of time. Intracellular and extracellular virions were collected by freezing and thawing 3 times, and plaque assay was performed to titrate the virus titer in the infected Vero and MDBK cells. The $y$-axis represents the virus titer, expressed as plaque-forming units per $0.1 \mathrm{~mL}$. (B) Cell apoptosis levels in Vero and MDBK cells infected with various serial virus doses for $48 \mathrm{~h}$. (C) Apoptosis levels in cells infected with 2 moi BEFV for the indicated length of time. Apoptotic ELISA was performed to quantify the extent of apoptosis. The $y$-axis represents the enrichment factor. Data are the means \pm S.D. of three individual experiments, each performed in duplicate. ${ }^{*} p<0.05$ compared with control cells. 

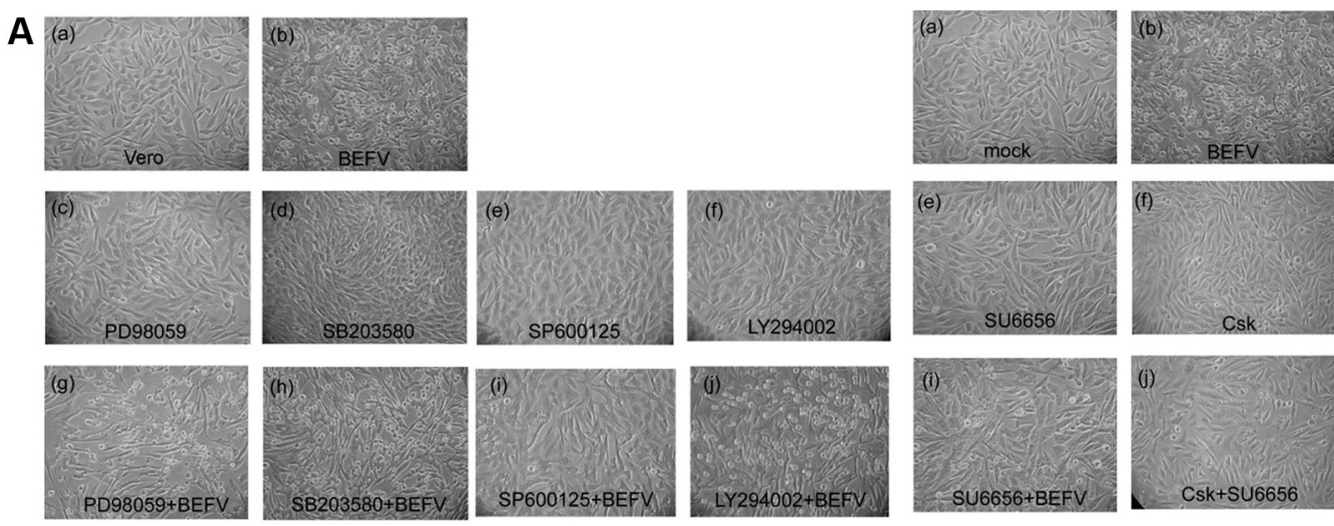
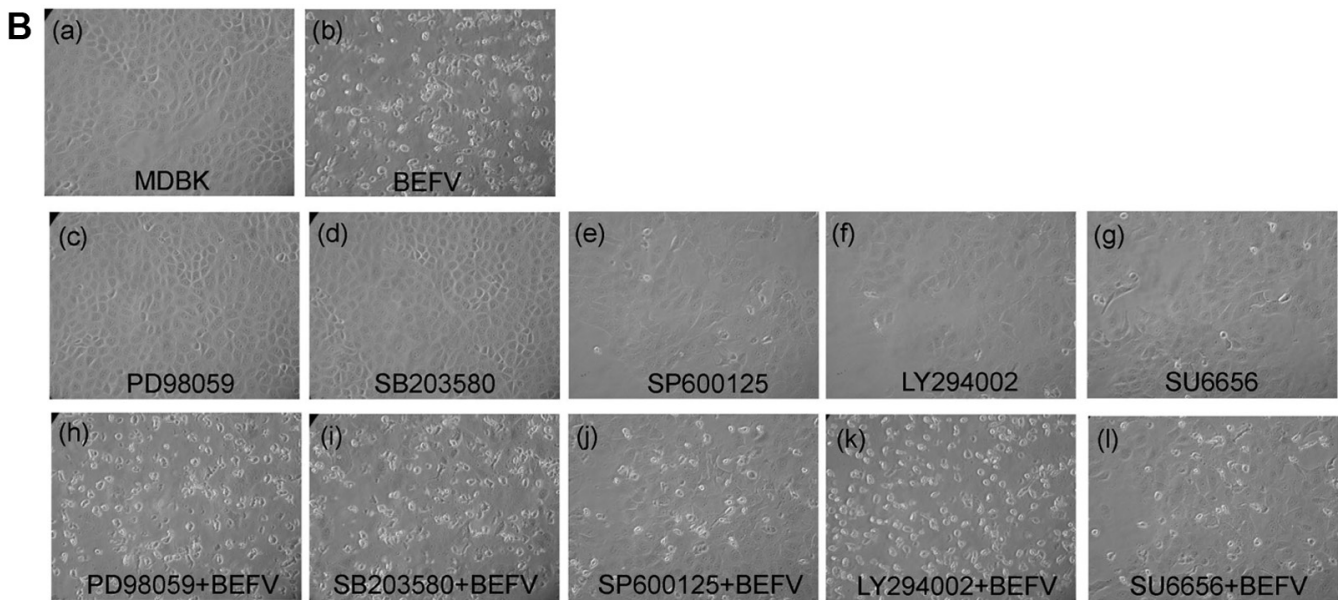

Figure 2. SP600125 and SU6656 released BEFV-mediated CPE. (A) Vero cells and (B) MDBK cells were pre-treated with the indicated inhibitors for $4 \mathrm{~h}$, then infected with 2 moi BEFV (A) or 5 moi BEFV (B) and incubated for an additional $48 \mathrm{~h}$. Cell images were obtained at a magnification of $200 \times$.

Specific antibodies against cleaved executioner caspase-3, -6 and -7, initiator caspase-9, the critical cellular substrate Poly (ADP-ribose) polymerase (PARP), and DNA fragmentation factor 45 (DFF45) were used to characterize the signaling cascade in apoptosis following BEFV infection. The levels of cleaved products of caspase-3, -6, and PARP were observed to gradually increase in infected Vero cells (Fig. 5A) and MDBK cells (Fig. 5B), although the appearance of the cleaved products was delayed in MDBK cells. Cytosol and mitochondria extracts were collected from BEFVinfected cells and the potential involvement of cytochrome c and Smac/DIABLO was further investigated. In Vero cells infected with 2 moi BEFV and MDBK cells infected with 5 moi $\mathrm{BEFV}$, cytochrome c was released from mitochondria in a time-dependent manner (Fig. 5C), whereas no effect was observed with Smac/DIBLO (Fig. 5C) or apoptosis-inducing factor (AIF) (data not shown), which is another key apoptosis-promoting factor within mitochondria that has been shown to participate in caspase-independent apoptosis in other studies. To further clarify the relationship between Src, $\mathrm{JNK}$, and these apoptosis effectors, MDBK cells pre-treated with SU6656 and SP600125 
Table I. SP600125 and SU6656 alleviated BEFV-mediated apoptosis.

\begin{tabular}{|c|c|c|c|c|c|c|}
\hline & \multicolumn{3}{|c|}{ Vero } & \multicolumn{3}{|c|}{ MDBK } \\
\hline & $\begin{array}{l}\text { Flow cytometry } \\
(\% \text { subG1) }\end{array}$ & $\begin{array}{l}\text { Apoptotic ELISA } \\
\text { (enrichment factor) }\end{array}$ & $\begin{array}{c}\text { MTT } \\
\text { (\% viability) }\end{array}$ & $\begin{array}{l}\text { Flow cytometry } \\
(\% \text { subG1) }\end{array}$ & $\begin{array}{l}\text { Apoptotic ELISA } \\
\text { (enrichment factor) }\end{array}$ & $\begin{array}{c}\text { MTT } \\
\text { (\% viability) }\end{array}$ \\
\hline Mock & $2.2 \pm 0.15$ & 1 & 100 & $2.8 \pm 0.6$ & 1 & 100 \\
\hline BEFV 2 moi & $67.5 \pm 6.1$ & $8.92 \pm 1.42$ & $28.6 \pm 1.6$ & $23.3 \pm 2.4$ & $9.05 \pm 1.13$ & $40.6 \pm 3.5$ \\
\hline BEFV+PD98059 & $60.2 \pm 5.7$ & $7.45 \pm 2.12$ & $25.4 \pm 2.6$ & $21.1 \pm 2.1$ & $7.21 \pm 1.24$ & $41.6 \pm 2.9$ \\
\hline $\mathrm{BEFV}+\mathrm{SB} 203580$ & $63.3 \pm 5.3$ & $8.08 \pm 1.63$ & $31.2 \pm 2.7$ & $19.9 \pm 1.7$ & $7.68 \pm 1.41$ & $39.1 \pm 3.1$ \\
\hline $\mathrm{BEFV}+\mathrm{SP} 600125$ & $23.5 \pm 3.5$ & $2.85 \pm 0.91^{*}$ & $76.2 \pm 4.6^{*}$ & $8.6 \pm 1.9$ & $2.35 \pm 0.82 *$ & $80.5 \pm 3.7^{*}$ \\
\hline BEFV+LY294002 & $59.9 \pm 5.4$ & $8.56 \pm 1.93$ & $32.2 \pm 2.2$ & $24.3 \pm 2.3$ & $7.07 \pm 0.93$ & $48.5 \pm 2.1$ \\
\hline $\mathrm{BEFV}+\mathrm{SU} 6656$ & $19.6 \pm 3.2$ & $2.54 \pm 0.82 *$ & $83.1 \pm 4.3^{*}$ & $7.8 \pm 1.7$ & $2.36 \pm 0.74^{*}$ & $78.6 \pm 5.7^{*}$ \\
\hline BEFV+SP600125+SU6656 & $18.3 \pm 2.8$ & $2.86 \pm 0.74 *$ & $85.7 \pm 3.9^{*}$ & $7.1 \pm 1.4$ & $2.00 \pm 0.81^{*}$ & $82.7 \pm 2.7^{*}$ \\
\hline
\end{tabular}

$* p<0.05$ compared with BEFV-infected cells. The rate of apoptotic ELISA is shown as the enrichment factor. MTT assay results are expressed as a percentage normalized against mock cells. 
A

SU6656

p-Src (Tyr 418)

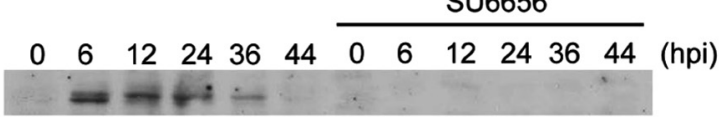

total Src

$\begin{array}{llllllllllll}1 & 2 & 3 & 4 & 5 & 6 & 7 & 8 & 9 & 10 & 11 & 12\end{array}$

B

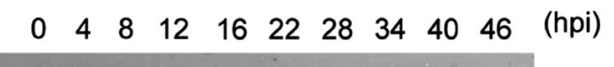

p-Src (Tyr 418$)$

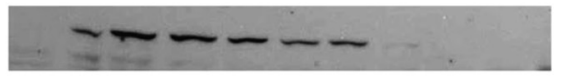

total Src

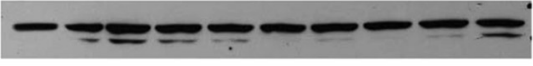

actin

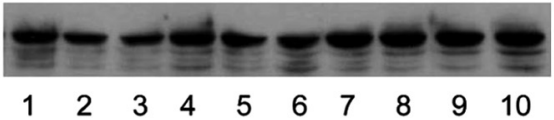

C

SU6656

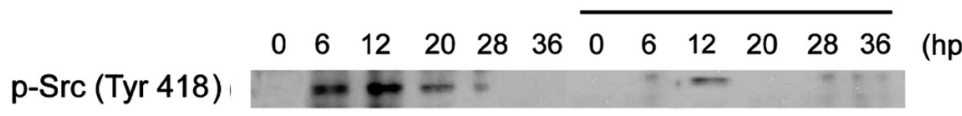

total Src

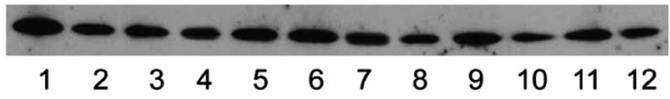

D

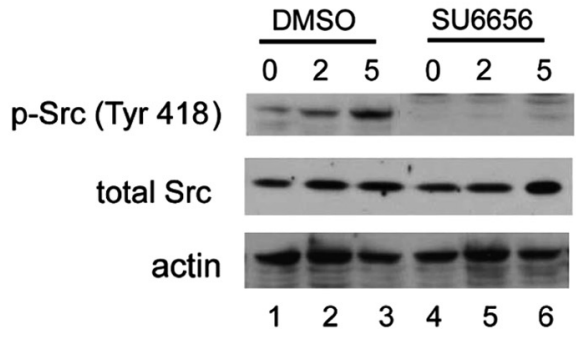

Figure 3. Up-regulation of Src phosphorylation level by BEFV. Vero cells were inoculated with 2 moi BEFV and incubated for the indicated length of time with or without SU6656 (A). MDBK cells were infected with 5 moi BEFV for the indicated length of time with (B) or without SU6656 (C). MDBK cells were infected with various doses of BEFV and incubated for an additional $24 \mathrm{~h}$ (D). A total of $30 \mu \mathrm{g}$ cell extract protein of each sample was loaded onto SDS-PAGE and subjected to Western blotting analysis.

were infected with BEFV then analyzed by Western blotting using the indicated antibodies. Under Src inhibition conditions, the levels of Src and JNK phosphorylation, cleaved products of caspase-3, -9, PARP, and DFF45 were all suppressed significantly; however, the levels of caspase- 6 and -7 were unchanged (Fig. 6A). Figure 6B shows that in the presence of SP600125, the phosphorylation level of Src was unchanged; however, the BEFV-induced JNK phosphorylation level and the cleaved product levels of caspase-3, -9, PARP, and DFF45 were all reduced. The levels of cleaved caspase- 6 and -7 were unchanged during BEFV infection. Further, SU6656 and SP600125 treatment inhibited cytochrome c release from 


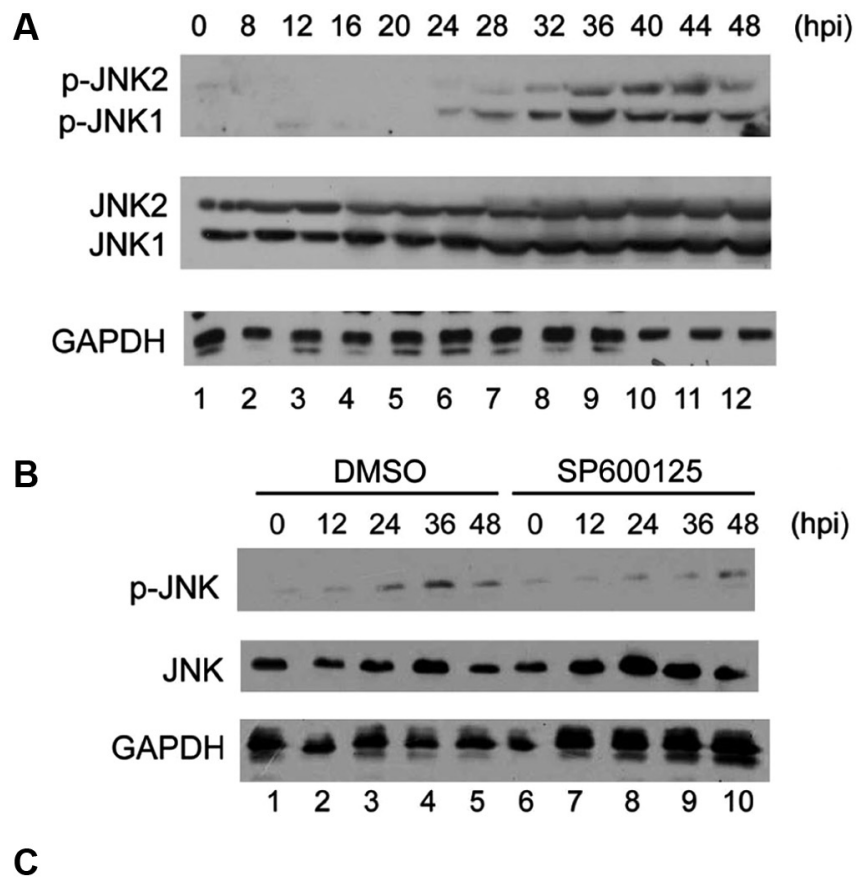

Vero

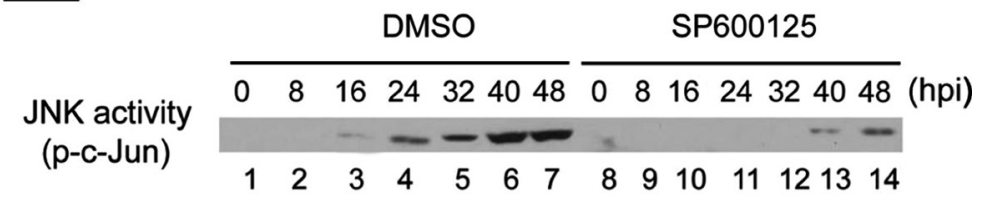

$\underline{\text { MDBK }}$

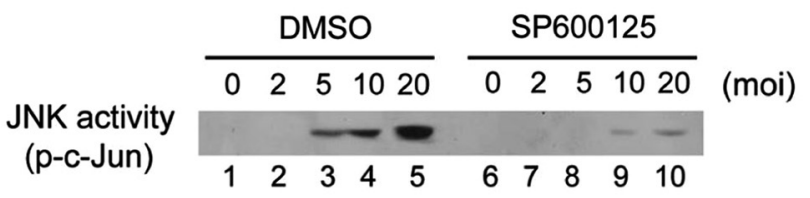

Figure 4. BEFV infection induced JNK phosphorylation and JNK activity. (A) Vero cells infected with 2 moi BEFV and (B) MDBK cells infected with 5 moi BEFV were cultured for the indicated additional length of time, and JNK phosphorylation was detected using specific phosphorylated JUN (p-JNK) antibody. (C) JNK kinase activity assay. Cell lysates collected from Vero cells (upper) infected with 2 moi and MDBK cells (lower) infected with BEFV for $48 \mathrm{~h}$ were subjected to the JNK kinase assay using c-Jun as the substrate. SP600125 specifically reversed the JNK activity signals.

mitochondria in BEFV-infected MDBK cells (Fig. 6C). The same experiments performed in Vero cells produced identical results (data not shown).

\section{DISCUSSION}

Several previous studies have confirmed the critical apoptosis-inducing function of the 
A

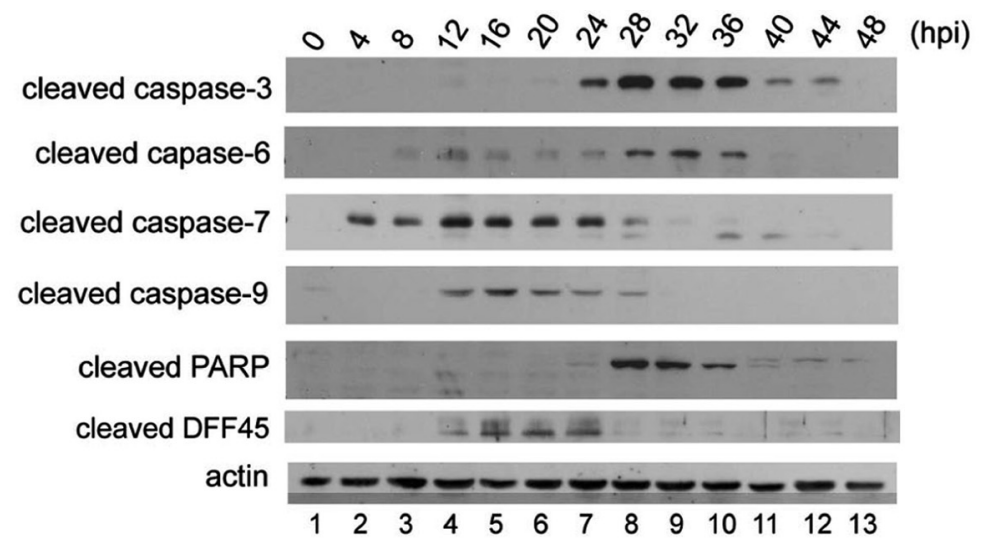

B

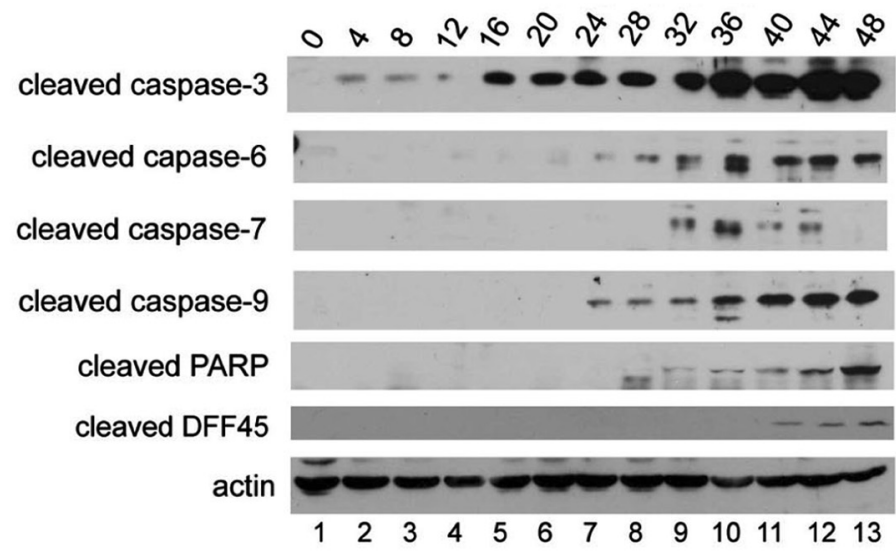

C

$\underline{\text { Vero }}$

$\underline{\text { MDBK }}$

BEFV 2 moi

$$
\frac{\text { cytosol }}{012243648} \frac{}{012243648}
$$

cyto $\mathrm{c}$

Smac/DIABLO

Cox IV

GAPDH

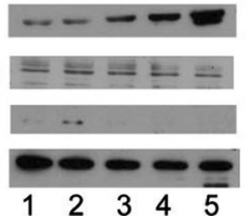

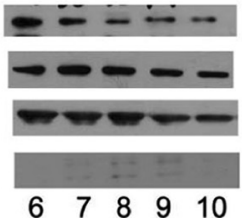

BEFV 5 moi

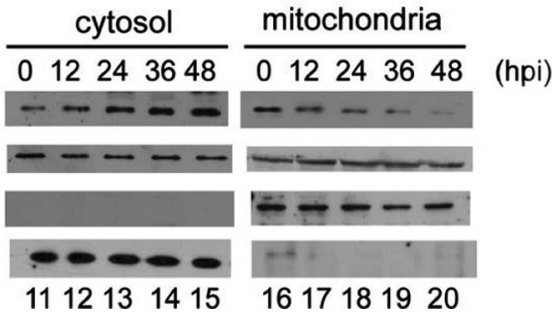

Figure 5. BEFV activated the caspase cascade and induced cytochrome c release from mitochondria. (A) Vero cells and (B) MDBK cells were infected with 2 or 5 moi BEFV respectively and incubate then subjected to Western blotting analysis. (C) Cytosol and mitochondria fractionation was performed as described in Materials and Methods Section. Cells were infected with BEFV for the indicated length of time, and equal amounts of cell extracts were subjected to Western blotting analysis. 
A

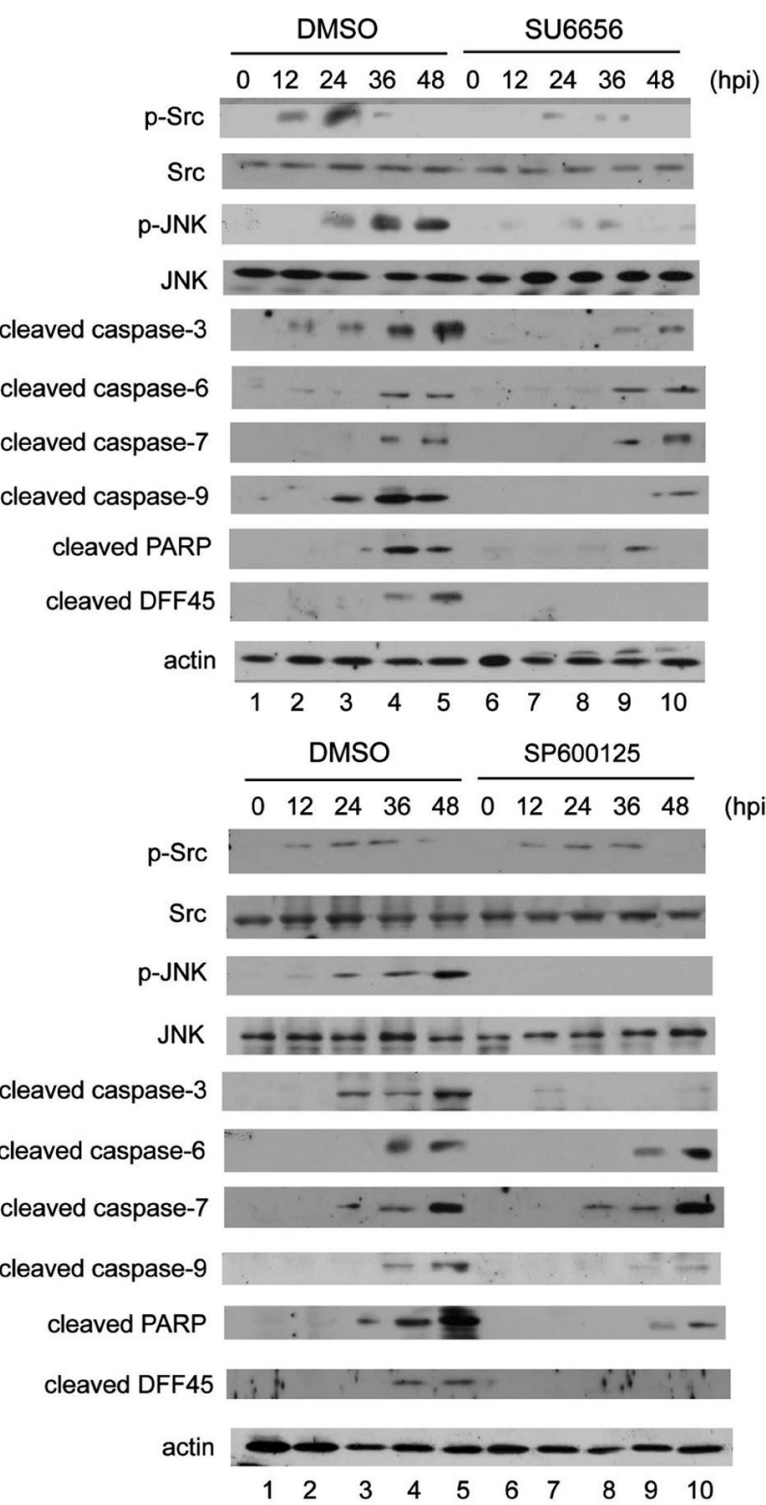

C
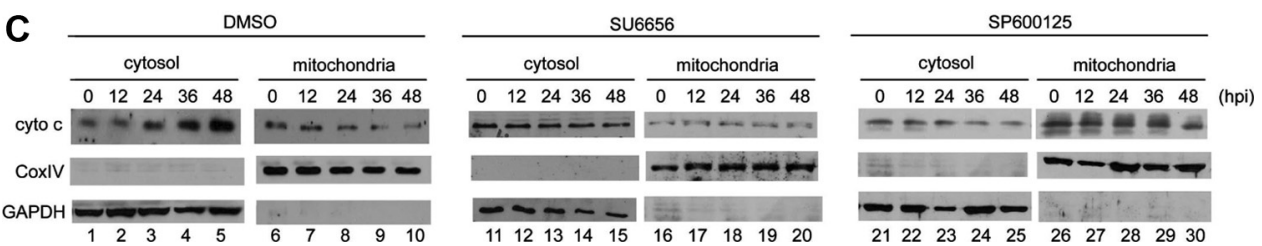

Figure 6. BEFV induced caspase cleavage through Src-JNK signaling. MDBK cells pre-treated with inhibitor or DMSO solvent for $4 \mathrm{~h}$ were then infected with BEFV. At the indicated time points, Western blotting (A and B) or cellular fractionation followed by Western blotting $(\mathrm{C})$ was performed. 
MAPK and Src tyrosine kinase pathways in a variety of experimental systems, especially in virus-infected cells $[13,17,29,33,41]$. We have previously shown that BEFV-induced apoptosis is caspase-dependent in a hamster fibroblast cell line, BHK-21 [5], and that the mitochondrial signaling pathway is involved [22]. In this study, we further investigated the molecular mechanism of BEFV-induced apoptosis in two different cell types. Our results demonstrated a higher efficiency of replication and a greater apoptosis induction ability of BEFV in Vero cells, and that BEFV triggers an apoptosis process that transiently induces a series of early response genes of $\mathrm{Src}$ and JNK. This leads to the release of cytochrome c to the cytosol from mitochondria, which is known to be the key step in the integration of cell death signals. In addition, the signaling further coordinates executioner caspase activation and causes cell death.

Our results show that Src and JNK were activated by BEFV infection: Src activation occurred at $6 \mathrm{hpi}$, and inhibition of Src with SU6656 blocked the loss of cell viability and increased the Sub G1 cell population, while activation of JNK presented later than Src activation, and inhibition of JNK with SP600125 also blocked apoptosis. It has been suggested that ERK1/ERK2 is generally associated with cell proliferation, and its activation is involved in apoptosis [40]. For example, inhibition of ERK1/ERK2 activation by PD98059 blocks oxidative stress- and radiation-induced apoptosis [6, 19], and also attenuates apoptosis induced by DNA damage stimuli [35]. It has been reported that p38 MAP kinase inhibitor SB203580 can attenuate hypoxia-induced apoptosis in osteoblast cells [4]. However, this involvement of ERK1/ERK2 and p38 was not observed in our study, which suggests that these molecules are not essential for BEFV-induced apoptosis. As shown in Table I and Figure 2, ERK1/ERK2 and p38 are not associated with BEFV-induced apoptosis; in contrast, cells treated with SU6656 and SP600125 released BEFV-mediated CPE, suggesting that the apoptosis is Src- and JNK-dependent.

In our previous study we demonstrated that BEFV induces significant DNA laddering and caspase-dependent apoptosis. We also showed that caspase inhibitor Z-VAD-fmk can block apoptosis [5]; however, direct biochemical analysis of the molecules has not yet been performed. In order to gain further insight into the mechanism of the signaling cascade, we performed an experiment to examine the molecular sequence of events in BEFV-induced apoptosis. The results obtained using inhibitors against Src or JNK revealed that upregulation of Src phosphorylation occurred prior to that of JNK phosphorylation. Inhibition of Src phosphorylation by SU6656 also downregulated JNK phosphorylation, suggesting that JNK is a downstream molecule in BEFV-induced cell death in Vero and MDBK cells.

Recent studies have revealed that caspases play a critical role in executing apoptosis [25]. We show that BEFV infection activates several caspases, including caspase-3, $-6,-7$, and -9 ; in addition, PARP and DFF45, downstream effectors of caspase, are also activated upon BEFV infection. In the activation of caspases by BEFV infection, only cleavage of caspase- 3 and -9 , as well as their downstream effectors PARP and DFF45, is regulated by an Srcdependent JNK signaling pathway. Cleaved PARP is a sign of developing apoptosis, since it is involved in DNA repair and responds to environmental stimuli [18]. Intact DFF45 is thought to be associated with increased DNA instability [16, 24]. The inhibition of caspase3 and -9 , and their downstream effectors, by Src and JNK inhibitors implies that JNK signaling is a critical pathway in BEFV-induced apoptosis, while other pathways regulating caspase- 6 and -7 also exist.

It is well-known that caspase cascade activation could depend on the release of apoptosispromoting factors by mitochondria such as cytochrome c [4, 10] and/or Smac/DIABLO $[1,26]$. Although Smac/DIABLO release from mitochondria is considered a general feature of UV-induced apoptosis [26], in our study we observed that the activation of the caspase cascade induced cytochrome c release, but no significant change in the Smac/DIABLO level was seen.

In summary, we report that BEFV infection induces Src-JNK signaling and increases 
cytochrome c release, which leads to the activation of caspase-3 and -9, with subsequent cleavage of PARP and DFF45. The molecular mechanism we have established in vitro in two different cell types reveals the participation of a signaling cascade in the BEFV infection process. We anticipate that further elucidation of the Src-JNK-regulated caspase cascade and other relevant mechanisms will provide knowledge applicable in the treatment of BEF.

Acknowledgements. We are very grateful to the editors of OxBiosci Editing Company for their valuable assistance in English editing and re-writing.

\section{REFERENCES}

[1] Adrain C., Creagh E.M., Martin S.J., Apoptosisassociated release of Smac/DIABLO from mitochondria requires active caspases and is blocked by Bcl-2, EMBO J. (2001) 20:6627-6636.

[2] Arias-Salgado E.G., Lizano S., Sarkar S., Brugge J.S., Ginsberg M.H., Shattil S.J., Src kinase activation by direct interaction with the integrin beta cytoplasmic domain, Proc. Natl. Acad. Sci. USA (2003) 100: 13298-13302.

[3] Campling B.G., Pym J., Baker H.M., Cole S.P., Lam Y.M., Chemosensitivity testing of small cell lung cancer using the MTT assay, Br. J. Cancer (1991) 63:75-83.

[4] Chae H.J., Kim S.C., Han K.S., Chae S.W., An N.H., Kim H.M., et al., Hypoxia induces apoptosis by caspase activation accompanying cytochrome $\mathrm{C}$ release from mitochondria in MC3T3E1 osteoblasts. p38 MAPK is related in hypoxia-induced apoptosis, Immunopharmacol. Immunotoxicol. (2001) 23:133-152.

[5] Chang C.J., Shih W.L., Yu F.L., Liao M.H., Liu H.J., Apoptosis induced by bovine ephemeral fever virus, J. Virol. Methods (2004) 122:165-170.

[6] Cho H.N., Lee Y.J., Cho C.K., Lee S.J., Lee Y.S., Downregulation of ERK2 is essential for the inhibition of radiation-induced cell death in HSP25 overexpressed L929 cells, Cell Death Differ. (2002) 9:448-456.

[7] Clarke P., Tyler K.L., Apoptosis in animal models of virus-induced disease, Nat. Rev. Microbiol. (2009) 7: 144-155.

[8] Desforges M., Despars G., Berard S., Gosselin M., McKenzie M.O., Lyles D.S., et al., Matrix protein mutations contribute to inefficient induction of apoptosis leading to persistent infection of human neural cells by vesicular stomatitis virus, Virology (2002) 295:63-73

[9] Du X.B., Lang J.Y., Xu J.R., Lu Y., Wen Y.J., Zhao J.M., et al., Vesicular stomatitis virus matrix protein gene enhances the antitumor effects of radiation via induction of apoptosis, Apoptosis (2008) 13:1205-1214.

[10] Finucane D.M., Bossy-Wetzel E., Waterhouse N.J., Cotter T.G., Green D.R., Bax-induced caspase activation and apoptosis via cytochrome $\mathrm{c}$ release from mitochondria is inhibitable by Bcl-xL, J. Biol. Chem. (1999) 274:22252233.

[11] Gaddy D.F., Lyles D.S., Vesicular stomatitis viruses expressing wild-type or mutant $M$ proteins activate apoptosis through distinct pathways, J. Virol. (2005) 79:4170-4179.

[12] Gaddy D.F., Lyles D.S., Oncolytic vesicular stomatitis virus induces apoptosis via signaling through PKR Fas, and Daxx, J. Virol. (2007) 81:2792-2804.

[13] Gillis P.A., Okagaki L.H., Rice S.A., Herpes simplex virus type 1 ICP27 induces p38 mitogen-activated protein kinase signaling and apoptosis in HeLa cells, J. Virol. (2009) 83:1767-1777.

[14] Hobbs J.A., Hommel-Berrey G., Brahmi Z., Requirement of caspase-3 for efficient apoptosis induction and caspase-7 activation but not viral replication or cell rounding in cells infected with vesicular stomatitis virus, Hum. Immunol. (2003) 64:82-92.

[15] Hsieh Y.C., Chen S.H., Chou C.C., Ting L.J., Itakura C., Wang F.I., Bovine ephemeral fever in Taiwan (2001-2002), J. Vet. Med. Sci. (2005) 67:411-416.

[16] Iwasa M., Imamura Y., Noriki S., Nishi Y., Kato H., Fukuda M., Immunohistochemical detection of early-stage carcinogenesis of oral leukoplakia by increased DNAinstability and various malignancy markers, Eur. J. Histochem. (2001) 45:333-346.

[17] Kumar A., Manna S.K., Dhawan S., Aggarwal B.B., HIV-Tat protein activates c-Jun N-terminal kinase and activator protein-1, J. Immunol. (1998) 161:776-781.

[18] Lazebnik Y.A., Kaufmann S.H., Desnoyers S., Poirier G.G., Earnshaw W.C., Cleavage of poly(ADPribose) polymerase by a proteinase with properties like ICE, Nature (1994) 371:346-347.

[19] Lee Y.J., Cho H.N., Soh J.W., Jhon G.J., Cho C.K., Chung H.Y., et al., Oxidative stress-induced apoptosis is mediated by ERK1/2 phosphorylation, Exp. Cell Res. (2003) 291:251-266.

[20] Liao Y.K., Inaba Y., Li N.J., Chain C.Y., Lee S.L., Liou P.P., Epidemiology of bovine ephemeral fever virus infection in Taiwan, Microbiol. Res. (1998) 153:289-295.

[21] Licata J.M., Harty R.N., Rhabdoviruses and apoptosis, Int. Rev. Immunol. (2003) 22:451-476.

[22] Lin C.H., Shih W.L., Lin F.L., Hsieh Y.C., Kuo Y.R., Liao M.H., Liu H.J., Bovine ephemeral fever virusinduced apoptosis requires virus gene expression and activation of Fas and mitochondrial signaling pathway, Apoptosis (2009) 864-877. 
[23] Lin P.Y., Lee J.W., Liao M.H., Hsu H.Y., Chiu S.J., Liu H.J., Shih W.L., Modulation of p53 by mitogenactivated protein kinase pathways and protein kinase $\mathrm{C}$ delta during avian reovirus $\mathrm{S} 1133$-induced apoptosis, Virology (2009) 385:323-334.

[24] Liu X., Li P., Widlak P., Zou H., Luo X., Garrard W.T., Wang X., The $40-\mathrm{kDa}$ subunit of DNA fragmentation factor induces DNA fragmentation and chromatin condensation during apoptosis, Proc. Natl. Acad. Sci. USA (1998) 95:8461-8466.

[25] Logue S.E., Martin S.J., Caspase activation cascades in apoptosis, Biochem. Soc. Trans. (2008) 36:1-9.

[26] McNeish I.A., Bell S., McKay T., Tenev T., Marani M., Lemoine N.R., Expression of Smac/DIABLO in ovarian carcinoma cells induces apoptosis via a caspase-9mediated pathway, Exp. Cell Res. (2003) 286:186-198.

[27] Megyeri K., Orosz L., Kemeny L., Vesicular stomatitis virus infection triggers apoptosis associated with decreased DeltaNp63alpha and increased Bax levels in the immortalized HaCaT keratinocyte cell line, Biomed. Pharmacother. (2007) 61:254-260.

[28] Nandi S., Negi B.S., Bovine ephemeral fever: a review, Comp. Immunol. Microbiol. Infect. Dis. (1999) 22:81-91.

[29] Ping-Yuan L., Hung-Jen L., Meng-Jiun L., FengLing Y., Hsue-Yin H., Jeng-Woei L., Wen-Ling S., Avian reovirus activates a novel proapoptotic signal by linking Src to p53, Apoptosis (2006) 11:2179-2193.

[30] Rice D.C., Dobrian A.D., Schriver S.D., Prewitt R.L., Src autophosphorylation is an early event in pressure-mediated signaling pathways in isolated resistance arteries, Hypertension (2002) 39:502-507.

[31] Schache P., Gurlevik E., Struver N., Woller N., Malek N., Zender L., et al., VSV virotherapy improves chemotherapy by triggering apoptosis due to proteasomal degradation of Mcl-1, Gene Ther. (2009) 16:849-861.

[32] Shi W., Tang Q., Chen X., Cheng P., Jiang P., Jing $X$., et al., Antitumor and antimetastatic activities of vesicular stomatitis virus matrix protein in a murine model of breast cancer, J. Mol. Med. (2009) 87:493-506.
[33] Singh I.N., El-Hage N., Campbell M.E., Lutz S.E., Knapp P.E., Nath A., Hauser K.F., Differential involvement of p38 and JNK MAP kinases in HIV-1 Tat and gp120-induced apoptosis and neurite degeneration in striatal neurons, Neuroscience (2005) 135:781-790.

[34] Snowdon W.A., Bovine ephemeral fever: the reaction of cattle to different strains of ephemeral fever virus and the antigenic comparison of two strains of virus, Aust. Vet. J. (1970) 46:258-266.

[35] Tang D., Wu D., Hirao A., Lahti J.M., Liu L., Mazza B., et al., ERK activation mediates cell cycle arrest and apoptosis after DNA damage independently of p53, J. Biol. Chem. (2002) 277:12710-12717.

[36] Tzipori S., Plaque assay and characteristics of strains of bovine ephemeral fever virus in Vero cells, $\mathrm{Br}$. Vet. J. (1975) 131:720-786.

[37] Uren M.F., St George T.D., Zakrzewski H., The effect of anti-inflammatory agents on the clinical expression of bovine ephemeral fever, Vet. Microbiol. (1989) 19:99-111.

[38] Walker P.J., Byrne K.A., Cybinski D.H., Doolan D.L., Wang Y.H., Proteins of bovine ephemeral fever virus, J. Gen. Virol. (1991) 72:67-74.

[39] Wang F.I., Hsu A.M., Huang K.J., Bovine ephemeral fever in Taiwan, J. Vet. Diagn. Invest. (2001) 13:462467.

[40] Xia Z., Dickens M., Raingeaud J., Davis R.J., Greenberg M.E., Opposing effects of ERK and JNK-p38 MAP kinases on apoptosis, Science (1995) 270:13261331.

[41] Yeh C.J., Lin P.Y., Liao M.H., Liu H.J., Lee J.W., Chiu S.J., et al., TNF-alpha mediates pseudorabies virusinduced apoptosis via the activation of p38 MAPK and JNK/SAPK signaling, Virology (2008) 381:55-66.

[42] Zhao J.M., Wen Y.J., Li Q., Wang Y.S., Wu H.B., $\mathrm{Xu}$ J.R., et al., A promising cancer gene therapy agent based on the matrix protein of vesicular stomatitis virus, FASEB J. (2008) 22:4272-4280. 